\title{
CORRESPONDENCE
}

\section{RE: Breast Cancer Risk After Salpingo-Oophorectomy in Healthy BRCA1/2 Mutation Carriers: Revisiting the Evidence for Risk Reduction}

\author{
Xinglei Chai, Susan Domchek, Noah Kauff, Timothy Rebbeck, Jinbo Chen
}

Affiliations of authors: Center for Clinical Epidemiology and Biostatistics (XC, TR, JC), Basser Center for BRCA (SD, TR), Abramson Cancer Center (SD, TR), and Division of Hematology-Oncology, Department of Medicine (SD), University of Pennsylvania School of Medicine, Philadelphia, PA; Clinical Genetics Service, Department of Medicine and Gynecology Service, Department of Surgery, Memorial Sloan-Kettering Cancer Center, New York, NY (NK).

Correspondence to: Jinbo Chen, PhD, Department of Biostatistics and Epidemiology, University of Pennsylvania Perelman School of Medicine, 203 Blockley Hall, 423 Guardian Drive, Philadelphia, PA 19104 (e-mail: jinboche@mail.med.upenn.edu) or Timothy Rebbeck, PhD, Department of Biostatistics and Epidemiology, University of Pennsylvania Perelman School of Medicine, 203 Blockley Hall, 423 Guardian Drive, Philadelphia, PA 19104 (e-mail: rebbeck@exchange.upenn.edu).

Heemskerk-Gerritsen et al. (1) questioned the protective effect of risk-reducing salpingo-oophorectomy (RRSO) on breast cancer (BC) risk in women who carry a BRCA1 or BRCA2 (BRCA1/2) mutation, as previously reported. They proposed that studies addressing this question should ensure: 1) women are eligible only if they do not have breast or ovarian cancer and have both ovaries and breasts intact before DNA testing; 2) each woman is followed from the age of DNA testing; and 3) the time at risk considers RRSO as a time-dependent variable that takes value 0 before RRSO and 1 after. Using this design, their analysis of Hereditary Breast and Ovarian Cancer in the Netherlands (HEBON) data reported a BC hazard ratio (HR) by RRSO of 1.09 (95\% confidence interval $[\mathrm{CI}]=0.67$ to 1.77 ). They hypothesized that the previously reported protective effect of RRSO on BC was because of failure to conform to one of these three design features.

Domchek et al. (2) and Kauff et al. (3) both have reported a protective effect of RRSO on BC for BRCA1/2 mutation carriers (Table 1) in women followed prospectively from ascertainment with breasts and ovaries intact at start of follow-up. These analyses followed women who underwent RRSO from RRSO date. Therefore, these analyses did not conform to feature 2 above and may be subject to "immortal person-time bias." We updated the Prevention and Observation of Surgical Endpoints (PROSE) dataset reported by Domchek to accommodate additional subjects recruited since the original publication and current followup. We then reanalyzed the PROSE and Kauff data using RRSO as a time-dependent variable following each woman from the age at ascertainment, as per Wacholder et al. (4).

The hazard ratio estimates in the current reanalyses were similar to those previously reported (Table 1): RRSO conferred a protective effect on BC in both the PROSE and Kauff data. In PROSE, all BC cases occurred more than five months following RRSO. In Kauff, BC occurring less than six months after RRSO were excluded. Thus, as in the paper of Heemskerk-Gerritsen, it is doubtful that cases unlikely to be influenced by RRSO biased either analysis. Mavaddat et al. (5) also reported hazard ratio estimates for RRSO using the same approach as HeemskerkGerritsen and found hazard ratios of $0.52(95 \% \mathrm{CI}=0.24$ to 1.13$)$ in BRCA1 and 0.79 (95\% CI $=0.35$ to 1.80 ) in BRCA2.

In Figure 2, Heemskerk-Gerritsen et al. displayed cumulative $\mathrm{BC}$ risk by RRSO based on a "landmark" method (6). The $\mathrm{x}$-axis of this figure started from age 30 years and risk started to increase at about age 31 years, implying the "landmark time" used was approximately 31 years. As the minimum age at RRSO in HEBON was 31 years, it is likely the estimated curve of the RRSO group was unstable when the number of carriers undergoing RRSO was small at the beginning of the curve. Choice of landmark time can clearly have large impact on the graphical display of the RRSO protective effect (6). It is not clear how the figure would appear if a different landmark time were used. Finally, if RRSO effects depend on age, then the difference in censoring events in HEBON vs the PROSE or Kauff data may lead to different effect estimates.

\section{References}

1. Heemskerk-Gerritsen BA, Seynaeve C, van Asperen CJ, et al. Breast cancer risk after salpingo-oophorectomy in healthy BRCA1/2 mutation carriers: revisiting the evidence for risk reduction. J Natl Cancer Inst. 2015;107(5):djv033 doi:10.1093/jnci/djv033.

2. Domchek SM, Rebbeck TR. Preventive surgery is associated with reduced cancer risk and mortality in women with BRCA1 and BRCA2 mutations. LDI issue brief. 2010;16(2):1-4.

3. Kauff N, Domchek S, Friebel T, et al. Risk-Reducing Salpingo-Oophorectomy for the Prevention of BRCA1 and BRCA2 Associated Breast and Gynecologic Cancer: A Multi-Center, Prospective Study. J Clin Oncol. 2008;26(8):1331-1337.

4. Wacholder S. Bias in intervention studies that enroll patients from high-risk clinics. J Natl Cancer Inst. 2004;96(16):1204-1207.

5. Mavaddat N, Peock S, Frost D, et al. Cancer Risks for BRCA1 and BRCA2 Mutation Carriers: Results From Prospective Analysis of EMBRACE. J Natl Cancer Inst. 2013;105(11):812-822.

6. Feuer EJ, Hankey BF, Gaynor JJ, et al. Graphical representation of survival curves associated with a binary non-reversible time dependent covariate. Stat Med. 1992;11(4):455-474.

7. Lin DY, Wei LJ. The robust inference for the Cox proportional hazards model. JASA. 1989;84:1074-1078. 
Table 1. Hazard ratio estimates of RRSO for breast cancer from the prospective cohort studies by Domchek et al. (2) and Kauff et al. (3) among BRCA1/2 carrier women without prior breast or ovarian cancer and without prior RRSO/RRM

\begin{tabular}{|c|c|c|c|}
\hline Study & BRCA1 & BRCA2 & BRCA1/2 \\
\hline Domchek et al. (2010), No. (\%) & $(n=970)$ & $(n=597)$ & $(\mathrm{n}=1567)$ \\
\hline \multicolumn{4}{|l|}{ RRSO } \\
\hline Yes & $294(30.3)$ & $155(26.0)$ & $449(28.7)$ \\
\hline Cancer & $40(13.6)$ & $13(8.4)$ & $53(11.8)$ \\
\hline No & $676(69.7)$ & $442(74.0)$ & $1118(71.3)$ \\
\hline Cancer & $137(20.3)$ & 96 (21.7) & $233(20.8)$ \\
\hline \multirow{3}{*}{$\begin{array}{l}\text { HR estimates }(95 \% \mathrm{CI}) \text { and } P \text { value using the approach of } \\
\text { Heemskerk-Gerritsen }(1)^{*}\end{array}$} & 0.63 & 0.40 & 0.59 \\
\hline & $(0.42 \text { to } 0.93)^{*}$ & $(0.19 \text { to } 0.84)^{*}$ & $(0.42 \text { to } 0.82)^{*}$ \\
\hline & $P=.021$ & $P=.015$ & $P=.001$ \\
\hline HR estimates $(95 \% \mathrm{CI})$ and $P$ value using & 0.56 & 0.42 & 0.51 \\
\hline \multirow[t]{2}{*}{ Domchek (2) original approach $†$} & $(0.39 \text { to } 0.81)^{*}$ & $(0.21 \text { to } 0.86)^{*}$ & $(0.36 \text { to } 0.70)^{*}$ \\
\hline & $P=.002$ & $P=.02$ & $P=.001$ \\
\hline Kauff et al. (2008), No. (\%) & $(n=220)$ & $(\mathrm{n}=125)$ & $(\mathrm{n}=345)$ \\
\hline \multicolumn{4}{|l|}{ RRSO } \\
\hline Yes & $100(45.5)$ & $60(48)$ & $160(46.4)$ \\
\hline Cancer & $6(6)$ & $2(3.3)$ & $8(5)$ \\
\hline No & $120(54.5)$ & $65(52)$ & $185(53.6)$ \\
\hline Cancer & $11(9.2)$ & $5(7.7)$ & $16(8.6)$ \\
\hline \multirow{3}{*}{ 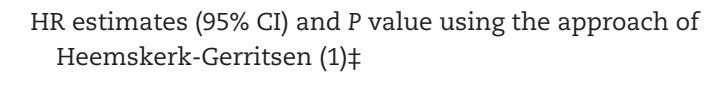 } & 0.47 & 0.47 & 0.50 \\
\hline & (0.16 to 1.37$)$ & (0.06 to 3.86$)$ & (0.20 to 1.25$)$ \\
\hline & $P=.17$ & $P=.49$ & $P=.14$ \\
\hline \multirow[t]{3}{*}{ Original HR estimates (95\% CI) and P value in Kauff (3) } & 0.61 & 0.28 & 0.53 \\
\hline & (0.30 to 1.22$)$ & (0.08 to 0.92$)$ & (0.29 to 0.96$)$ \\
\hline & $P=.16$ & $P=.036$ & $P=.036$ \\
\hline
\end{tabular}

${ }^{*}$ Age was used as the time scale with left truncation at the age of ascertainment in the Cox regression analyses. RRSO was used as a time-dependent variable that took value 0 before and 1 after RRSO. We stratified on study center and accounted for family correlation using robust variance estimates (7). All analyses adjusted for year of birth (<1940, 1940-49, 1950-59, 1960-69, $\geq 1970)$, parity (yes or no), and, in the analyses of combined carrier group, mutation status (BRCA1 or BRCA2). For BRCA2, year of birth was adjusted as whether born before 1960 or not. RRSO = risk-reducing salpingo-oophorectomy.

† This analysis used the same approach as Domchek et al. (2010). Time since age of RRSO was used for RRSO users, and time since ascertainment was used for non-RRSO users as time scale in Cox regression analyses. We stratified on study center and accounted for family correlation using robust variance estimates (7). All analyses adjusted for year of birth (whether born before 1960 or not).

‡ The analysis was the same as in the first note, except that follow-up started from age at genetic testing. Parity (yes or no) was adjusted for BRCA1, history of prior use of hormone replacement therapy (yes or no) for BRCA2, and both for the combined carrier group in addition to the mutation status. 